\title{
Tendência das produções científicas brasileiras acerca do potencial terapêutico do
}

\section{Alecrim à saúde}

\author{
Trend of Brazilian scientific productions on the therapeutic potential of Rosemary for health \\ Tendencias de las producciones científicas brasileñas sobre el potencial terapéutico del Romero
}

para la salud

Recebido: 14/07/2021 | Revisado: 21/07/2021 | Aceito: 24/07/2021 | Publicado: 01/08/2021

Carolina Renz Pretto

ORCID: https://orcid.org/0000-0002-6925-7969

Universidade Federal de Santa Maria, Brasil

E-mail: carol.renzpretto@gmail.com

Juliana Tamiozzo

ORCID: https://orcid.org/0000-0003-3586-379X

Universidade Federal de Santa Maria, Brasil

E-mail: julianatamiozzo4@gmail.com

Eduardo Rodrigues Lauz

ORCID: https://orcid.org/0000-0001-8549-6751

Universidade Federal de Santa Maria, Brasil

E-mail: edulauz@yahoo.com.br

Christiane de Fátima Colet

ORCID: https://orcid.org/0000-0003-2023-5088

Universidade Regional do Noroeste do Estado do Rio Grande do Sul, Brasil

E-mail: chriscolet@yahoo.com.br

Carme Lúcia Colomé Beck

ORCID: https://orcid.org/0000-0001-9060-1923

Universidade Federal de Santa Maria, Brasil

E-mail: carmembeck@gmail.com

Rosângela Marion da Silva

ORCID: https://orcid.org/0000-0003-3978-9654

Universidade Federal de Santa Maria, Brasil

E-mail: cucasma@terra.com.br

\begin{abstract}
Resumo
Objetivo: analisar a tendência das produções científicas brasileiras acerca do potencial terapêutico do Alecrim à saúde Metodologia: trata-se de uma revisão integrativa da literatura com levantamento bibliográfico realizado de dezembro de 2020 a janeiro de 2021, na Biblioteca Digital de Teses e Dissertações: BDTD e no Catálogo de Teses e Dissertações (CAPES). Foram encontradas 682 produções das quais 71 foram analisadas. Dados coletado com uso de instrumento desenvolvido pelos autores e analisados a partir da identificação de semelhanças, diferenças e realização de inferências. Resultados: do total de produções $70,4 \%$ eram dissertações e $29,6 \%$ teses, a maioria proveniente de programas de pós-graduação em Ciências Farmacêuticas e Veterinária, 54,9\% pesquisas foram desenvolvidas in vitro, $35,2 \%$ in vivo (duas incluíram humanos). Quanto aos efeitos do Alecrim ou de seus compostos, $59,2 \%$ analisaram o efeito antimicrobiano e antibiofilme, $19,7 \%$ o anti-inflamatório, $16,9 \%$ a atividade antioxidante, $12,7 \%$ a viabilidade celular e citotoxicidade, entre outros. Pesquisas com o desenvolvimento de produtos somaram 15,5\%. Considerações Finais: há uma tendência crescente de produções científicas brasileiras acerca do potencial terapêutico do Alecrim à saúde, com envolvimento de diversas universidades do país. A falta de pesquisas provenientes de Programas de PósGraduação em Enfermagem abre portas para sua inserção na temática. Os estudos tenderam a avaliar a ação antimicrobiana e antibiofilme do Alecrim e seus compostos, seguido do efeito anti-inflamatório e antioxidante evidenciando potencial terapêutico contra microrganismos e para doenças crônicas. Mais pesquisas sobre o tema são necessárias, inclusive com vistas ao desenvolvimento de produtos.
\end{abstract}

Palavras-chave: Terapias complementares; Rosmarinus; Usos terapêuticos; Saúde.

\begin{abstract}
Objective: to analyze the trend of Brazilian scientific production about the therapeutic potential of Rosemary for health Methodology: this is an integrative literature review with a bibliographic survey carried out from December 2020 to January 2021, in the Digital Library of Theses and Dissertations: BDTD and in the Theses and Dissertations Catalog (CAPES). 682 productions were found, of which 71 were analyzed. Data collected using an instrument developed by the authors and analyzed based on the identification of similarities, differences and inferences. Results:
\end{abstract}


$70.4 \%$ of the total productions were dissertations and $29.6 \%$ theses, most from graduate programs in Pharmaceutical Sciences and Veterinary, 54.9\% researches were developed in vitro, 35.2\% in vivo (two included humans). As for the effects of Rosemary or its compounds, 59.2\% analyzed the antimicrobial and antibiofilm effects, $19.7 \%$ the antiinflammatory, $16.9 \%$ the antioxidant activity, $12.7 \%$ the cell viability and cytotoxicity, among others. Product development surveys totaled $15.5 \%$. Final Considerations: there is a growing trend of Brazilian scientific production about the therapeutic potential of Rosemary for health, with the involvement of several universities in the country. The lack of research from Graduate Nursing Programs opens doors for their inclusion in the subject. The studies tended to evaluate the antimicrobial and antibiofilm action of Rosemary and its compounds, followed by the antiinflammatory and antioxidant effect, showing therapeutic potential against microorganisms and for chronic diseases. More research on the subject is needed, including with a view to product development.

Keywords: Complementary therapies; Rosmarinus; Therapeutic uses; Health.

\section{Resumen}

Objetivo: evaluar la tendencia de las producciones brasileñas sobre el potencial terapéutico del Romero a salud. Metodología: revisión integradora de la literatura con levantamiento bibliográfico realizado de diciembre de 2020 a enero de 2021, en la Biblioteca Digital de Teses e Dissertações: BDTD e en el Catálogo de Teses e Dissertações (CAPES). Fueron encontradas 682 producciones y evaluadas 7. Dados colectados con instrumento desarrollado por los autores y analizados por identificación de similitudes, diferencias e inferencias. Resultados: de las producciones, $70,4 \%$ eran disertaciones y $29,6 \%$ tesis, la mayoría de programas de postgrado en Ciencias Farmacéuticas y Veterinaria, $54,9 \%$ investigaciones desarrolladas in vitro, $35,2 \%$ in vivo (dos involucraron humanos). Cuanto los efectos del Romero o sus compuestos, 59,2\% analizaron el antimicrobiano y antibiofilm, 19,7\% el antinflamatorio, $16,9 \%$ la actividad antioxidante, $12,7 \%$ viabilidad celular y citotoxicidad, y otros. Investigaciones con desarrollo de productos sumaron $15,5 \%$. Consideraciones Finales: hay una tendencia creciente de producciones brasileñas sobre el potencial terapéutico del Romero a salud con involucramiento de muchas universidades nacionales. Faltan investigaciones de programas de postgrado en Enfermería y eso abre puertas a su inserción en el tema. Los estudios tenderán evaluar la acción antimicrobiana y antibiofilm del Romero y compuestos, el efecto desinflamante y antioxidante evidenciando propriedades contra microrganismos y enfermedades crónicas. Más investigaciones son necesarias, también con desarrollo de productos.

Palabras clave: Terapias complementarias; Rosmarinus; Usos terapéuticos; Salud.

\section{Introdução}

A medicina tradicional e complementar compreende um amplo conjunto de produtos e práticas profissionais baseados em conhecimentos, teorias, crenças e experiências de diferentes culturas para manter a saúde, prevenir, diagnosticar ou tratar doenças físicas ou mentais que não pertencem ou não são integradas totalmente à medicina convencional. Entre os produtos estão as ervas e outros produtos com princípios ativos de origem vegetal, mineral ou animal; as práticas compreendem medicamentos terapêuticos, tratamentos de saúde e procedimentos - naturopatia, acupuntura, terapias manuais, reiki, osteopatia, e outras terapias físicas, mentais e espirituais (OMS, 2019).

Desde a Declaração de Alma-Ata em 1978, a Organização Mundial da Saúde - OMS vem estimulando os estadosmembros à formulação de políticas nacionais para utilização de terapias tradicionais e complementares e o desenvolvimento de investigações sobre sua segurança, qualidade, eficácia e acesso, com intuito de responder à transição demográfica, epidemiológica e valorizar práticas populares de cuidado (Silva et al., 2020). No Brasil, a partir da década de 80 , com a $8^{\circ}$ Conferência Nacional de Saúde e implantação e desenvolvimento do Sistema Único de Saúde (SUS), começaram os esforços para a efetivação destas práticas na atenção à saúde e culminaram, em 2006, com a criação da Política Nacional de Práticas Integrativas e Complementares no SUS (PNPIC) e oferta de diferentes práticas no âmbito do SUS, entre as quais homeopatia, plantas medicinais e fitoterápicas (Mendes et al., 2019).

No mesmo ano, foi instituída a Política Nacional de Plantas Medicinais e Fitoterápicos com vistas à ampliação do acesso da população aos medicamentos, opções terapêuticas, melhoria da atenção, desenvolvimento industrial e tecnológico e uso sustentável da biodiversidade brasileira. Desde então, há um interesse crescente quanto ao uso terapêutico das plantas medicinais e fitoterápicos no país, embora os profissionais de saúde ainda careçam de conhecimento quanto às suas indicações de uso (Borges \& Sales, 2018). 
Entre as plantas medicinais, cita-se o Rosmarinus Officinalis (Alecrim), planta de origem mediterrânea, cultivada no mundo inteiro, inclusive no Brasil. Pertencente à família Lamiaceae, a planta tem sido tradicionalmente utilizada na culinária e também no tratamento de doenças. Constitui-se de polifenóis, di e triterpenos e óleos essenciais, que além de suas propriedades antioxidantes protegem a planta contra patógenos, motivos pelos quais ela é do interesse à saúde humana (Andrade et al., 2018).

Estudos têm demonstrado efeitos in vitro e/ou in vivo (principalmente ratos e camundongos), tanto do Alecrim integral como de compostos específicos, em diversas condições de saúde. Verificou-se efeito positivo na remodelação cardíaca após infarto, neuroproteção na isquemia cerebral, efeito antinociceptivo, redução de biofilmes, inibição de nefro e hepatotoxicidade provocada por medicamentos, efeito anti-hipertensivo, antidepressivo e inibição da proliferação de células tumorais (Oliveira et al., 2019). Ensaio clínico com estudantes universitários também evidenciou que cápsulas de folhas secas de Alecrim melhoram o desempenho da memória, sono e contribuíram para reduzir ansiedade e depressão (Nematolahi et al., 2018).

Diante dos possíveis benefícios terapêuticos do Alecrim, torna-se preponderante a verificação do estado atual do conhecimento sobre os seus usos medicinais, pois, a identificação das lacunas existentes pode colaborar com a construção do conhecimento e o aprofundamento das evidências existentes, importantes para o desenvolvimento de produtos e para inclusão desta planta ou fitoterápico no cuidado à saúde humana, inclusive pela enfermagem. Assim, este estudo tem como objetivo analisar a tendência das produções científicas brasileiras acerca do potencial terapêutico do Alecrim à saúde.

\section{Metodologia}

Trata-se de um estudo exploratório e descritivo do tipo revisão integrativa da literatura. Este método permite a sumarização da literatura existente sobre o tema investigado integrando diferentes metodologias e perspectivas, importante para definir conceitos, fazer generalizações, identificar as lacunas do conhecimento (Whittemore \& Knafl, 2005), as tendências de pesquisa no tema, e indicar os caminhos futuros das investigações. Assim, esta revisão foi desenvolvida seguindo as etapas: elaboração da questão de pesquisa; definição das bases de dados e critérios de inclusão e exclusão dos estudos; definição das informações a serem extraídas dos estudos; avaliação dos estudos incluídos na revisão; interpretação dos resultados; e apresentação da síntese do conhecimento (Whittemore \& Knafl, 2005).

Como pergunta de pesquisa foi definida: qual a tendência das produções científicas brasileiras acerca do potencial terapêutico do Alecrim à saúde? O levantamento bibliográfico ocorreu de dezembro de 2020 a janeiro de 2021, na Biblioteca Digital de Teses e Dissertações: BDTD e no Catálogo de Teses e Dissertações (CAPES). Para sua operacionalização foram testadas várias estratégias de busca nas bases de dados, contudo, verificou-se que uma estratégia mais simples e ampla, com menos palavras era mais eficaz para encontrar os estudos de interesse.

Na BDTD, com a estratégia "(Título:rosmarinus OU Título:"Rosmarinus Officinalis" OU Título:rosemary OU Título:alecrim)" foram identificadas 121 publicações. No Catálogo de Teses e Dissertações (CAPES) com a mesma estratégia foram identificados 561 documentos. Utilizou-se o software de gerenciamento de referências Mendeley para identificar duplicatas, ordenar e selecionar as publicações relevantes. Alguns documentos foram selecionados manualmente devido aos problemas de importação ou à forma como foi realizado o registro do mesmo na base. Pela escolha de considerar documentos em apenas uma base de dados, foram excluídos 14 estudos.

Para seleção das produções, foram estabelecidos como critérios de inclusão: teses ou dissertações brasileiras acerca do potencial terapêutico do Alecrim à saúde, nos idiomas inglês, português e espanhol. Os critérios de exclusão foram: estudos com resumo indisponível na base de dados e sem possibilidade de acesso por outras fontes na internet; pesquisas que versam sobre a utilização do Alecrim como conservante de alimentos ou efeito sobre contaminantes alimentares; propriedades do 
Alecrim no tratamento de doenças específicas de plantas ou animais; estudos com Alecrim combinado a outros produtos, sem possibilidade de verificar seu efeito em separado; estudos com resumos que não contém informações suficientes sobre o efeito do Alecrim e/ou dos seus constituintes, óleos ou extratos.

Foram incluídos na revisão 71 documentos, 21 da BDTD e 50 na CAPES. Para a coleta das informações dos documentos foi desenvolvido um instrumento constituído dos itens: título; local/ano; autor/orientador; programa; tipo de produção (tese ou dissertação); objetivo; metodologia; resultados principais; tendência futura. Todos os dados foram coletados a partir dos resumos das produções.

A análise crítica das produções compreendeu a leitura atenta dos resumos, identificação de semelhanças, diferenças, foco da investigação e outros aspectos. Os resultados foram apresentados de maneira descritiva, explicitados por meio de gráficos e tabelas para melhor compreensão pelo leitor e interpretados por meio de inferências, à luz da literatura.

Quanto aos aspectos éticos, por se tratar de uma revisão, o presente estudo não foi submetido ao comitê de ética, mas todas as ideias, definições e conceitos dos autores das produções analisadas foram respeitados de acordo com os princípios de citação de autoria.

\section{Resultados}

Do total de 71 produções encontradas, $50(70,4 \%)$ eram dissertações e $21(29,6 \%)$ teses. A primeira produção identificada foi em 2004 e o pico quantitativo em 2017, embora pode-se afirmar tendência crescente nas produções sobre o tema, conforme Figura 1.

Figura 1 - Número de produções/ano acerca do potencial terapêutico do Alecrim à saúde.

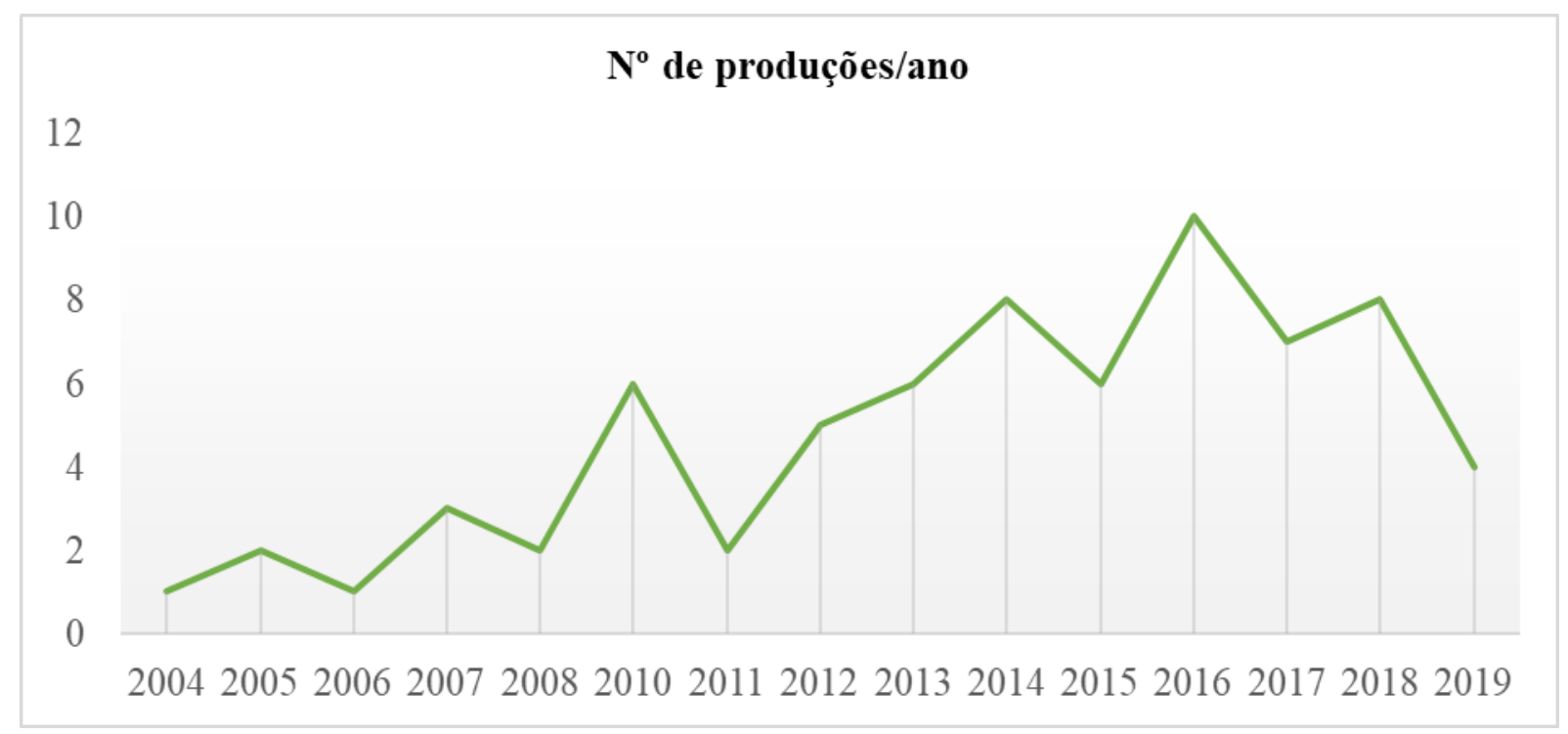

Fonte: Autores.

Em relação às universidades que mais investigam sobre o tema, merecem destaque a Universidade Federal de Pelotas e a Universidade Federal de Santa Maria, cada uma com oito pesquisas, e a Universidade Federal de Santa Catarina com seis, Tabela 1. 
Tabela 1 - Número de produções acerca do potencial terapêutico do Alecrim à saúde por universidade.

\begin{tabular}{|c|c|}
\hline Universidade & $\mathbf{N}^{0}$ produções (\%) \\
\hline Universidade Federal de Pelotas & $8(11,3)$ \\
\hline Universidade Federal de Santa Maria & $8(11,3)$ \\
\hline Universidade Federal de Santa Catarina & $6(8,5)$ \\
\hline Universidade Estadual de Maringá & $4(5,6)$ \\
\hline Universidade Estadual Paulista & $4(5,6)$ \\
\hline Universidade Federal de Pernambuco & $4(5,6)$ \\
\hline Universidade de São Paulo & $3(4,2)$ \\
\hline Universidade Federal de Goiás & $3(4,2)$ \\
\hline Universidade Estadual de Campinas & $2(2,8)$ \\
\hline Universidade Federal da Paraíba & $2(2,8)$ \\
\hline Universidade Federal de Lavras & $2(2,8)$ \\
\hline Universidade Federal do Amapá & $2(2,8)$ \\
\hline Universidade Federal do Espírito Santo & $2(2,8)$ \\
\hline Universidade Federal do Rio Grande do Sul & $2(2,8)$ \\
\hline Universidade Federal Fluminense & $2(2,8)$ \\
\hline Pontifícia Universidade Católica do Rio Grande do Sul & $1(1,4)$ \\
\hline Universidade de São Francisco & $1(1,4)$ \\
\hline Universidade de Brasília & $1(1,4)$ \\
\hline Universidade de Franca & $1(1,4)$ \\
\hline Universidade de Vila Velha & $1(1,4)$ \\
\hline Universidade Estadual de Londrina & $1(1,4)$ \\
\hline Universidade Federal de Juíz de Fora & $1(1,4)$ \\
\hline Universidade Federal do Acre & $1(1,4)$ \\
\hline Universidade Federal do Pará & $1(1,4)$ \\
\hline Universidade Federal do Paraná & $1(1,4)$ \\
\hline Universidade Federal do Piauí & $1(1,4)$ \\
\hline Universidade Federal do Rio de Janeiro & $1(1,4)$ \\
\hline Universidade Federal do Triângulo Mineiro & $1(1,4)$ \\
\hline Universidade Jose do Rosário Vellano & $1(1,4)$ \\
\hline Universidade Luterana do Brasil & $1(1,4)$ \\
\hline Universidade Metodista de São Paulo & $1(1,4)$ \\
\hline Universidade Tecnológica Federal do Paraná & $1(1,4)$ \\
\hline Total & $71(100)$ \\
\hline
\end{tabular}

Fonte: Autores.

No que se refere aos programas de pós-graduação com maior número de pesquisas sobre o potencial terapêutico do Alecrim, constata-se que o de Ciências Farmacêuticas e o da Veterinária são responsáveis por 15,5\% e 9,9\% das produções, respectivamente (Tabela 2). Nota-se que programas que envolvem algum ramo da farmácia (Ciências Farmacêuticas, Bioquímica e Farmacologia, Farmácia, Farmacologia, Inovação Farmacêutica) representam juntos 23,9\% das produções. 
Salienta-se que o Programa de Pós-Graduação em Veterinária da Universidade Federal de Pelotas respondeu por cinco investigações e na Universidade Federal de Santa Maria, os Programa de Pós-Graduação em Ciências Farmacêuticas e Programa de Pós-Graduação em Ciências Biológicas colaboraram com duas cada.

Tabela 2 - Proporção de produções dos programas de pós-graduação acerca do potencial terapêutico do Alecrim à saúde.

\begin{tabular}{lr}
\hline \multicolumn{1}{c}{ Programa } & $\mathbf{N}^{\mathbf{0}}$ de Produções $(\%)$ \\
\hline Ciências Farmacêuticas & $11(15,5)$ \\
Veterinária & $7(9,9)$ \\
Ciência dos Alimentos & $5(7,0)$ \\
Odontologia & $4(5,6)$ \\
Ciências Biológicas: Bioquímica Toxicológica & $3(4,2)$ \\
Neurociências & $3(4,2)$ \\
Ciências da Saúde & $2(2,8)$ \\
Biologia Buco-Dental & $2(2,8)$ \\
Biologia Celular e Molecular & $2(2,8)$ \\
Bioquímica e Farmacologia & $2(2,8)$ \\
Biotecnologia & $2(2,8)$ \\
Ciências & $2(2,8)$ \\
Ciências Aplicadas a Produtos para Saúde & $2(2,8)$ \\
Ciências Veterinárias & $2(2,8)$ \\
Farmácia & $2(2,8)$ \\
Química & $1(1,4)$ \\
Agroecossistema & $1(1,4)$ \\
Agronomia & $1(1,4)$ \\
Agroquímica & $1(1,4)$ \\
Biologia de Agentes Infecciosos e Parasitários & $1(1,4)$ \\
Biopatologia Bucal & $1(1,4)$ \\
Bioquímica e Bioprospecção & $1(1,4)$ \\
Ciência Animal & $1(1,4)$ \\
Ciências Biológicas & $1(1,4)$ \\
Ciências da Saúde na Amazônia & $1(1,4)$ \\
Engenharia e Ciência de Alimentos & $1(1,4)$ \\
Engenharia e Tecnologia de Materiais & $1(1,4)$ \\
Farmacologia & $1(1,4)$ \\
Fisiopatologia em Clínica Médica & $1(1,4)$ \\
Genética e Toxicologia Aplicada & $1(1,4)$ \\
Inovação Farmacêutica & $1(1,4)$ \\
Inovação Tecnológica & $1(1,4)$ \\
Produtos Naturais e Sintéticos Bioativos & $1(1,4)$ \\
Psicologia da Saúde & $1(1,4)$ \\
Tootecnia & $1(1,4)$ \\
\hline & $71(100)$ \\
\hline
\end{tabular}

Fonte: Autores.

Mais da metade das pesquisas com o Alecrim e/ou seus componentes, 39 (54,9\%), foi desenvolvida in vitro, 25 $(35,2 \%)$ in vivo e $8(11,3 \%)$ envolveram ambas as formas. Os estudos foram realizados com diversos tipos de microrganismos, entre eles bactérias, vírus e fungos, e diferentes linhagens celulares. Entre os estudos in vivo, dois envolveram humanos $(8,0 \%)$, os demais, 22 , incluíram ratos ou camundongos $(88,0 \%)$ e um deles um nematódeo $(4,0 \%)$. 
Quanto a ação do Alecrim ou de seus compostos avaliada nas investigações, 59,2\% analisaram o efeito antimicrobiano e antibiofilme, $19,7 \%$ o efeito anti-inflamatório, $16,9 \%$ a atividade antioxidante, $12,7 \%$ a viabilidade celular e citotoxicidade, $7,0 \%$ o potencial antidepressivo, 4,2\% o efeito antinociceptivo, o efeito gastroprotetor, a neuroproteção ou efeito na ansiedade ou transtorno obsessivo compulsivo, os demais investigaram outras questões (Quadro 1). Destaca-se que boa parte dos estudos apresentaram mais de um objetivo.

Quadro 1 - Tipo de ação do Alecrim ou de seus compostos avaliada nas produções.

\begin{tabular}{|l|r|}
\hline \multicolumn{1}{|c|}{ Tipo de ação avaliada } & \multicolumn{1}{c|}{ No de Produção (\%) $^{-1}$} \\
\hline Efeito antimicrobiano e antibiofilme & $42(59,2)$ \\
\hline Efeito anti-inflamatório & $14(19,7)$ \\
\hline Atividade antioxidante/marcadores de estresse oxidativo & $12(16,9)$ \\
\hline Avaliar viabilidade celular- citotoxicidade & $9(12,7)$ \\
\hline Potencial antidepressivo & $5(7,0)$ \\
\hline Efeito antinocioceptivo & $3(4,2)$ \\
\hline Efeito gastroprotetor & $3(4,2)$ \\
\hline Efeito neuroprotetor & $3(4,2)$ \\
\hline Efeito na ansiedade ou transtorno obsessivo & $3(4,2)$ \\
\hline Efeito em perfil lipídico & $2(2,8)$ \\
\hline Efeito na aprendizagem e memória & $2(2,8)$ \\
\hline Efeito na atividade motora & $2(2,8)$ \\
\hline Fotoproteção & $2(2,8)$ \\
\hline Genotoxicidade & $1(1,4)$ \\
\hline Motilidade Gástrica - reduz & $1(1,4)$ \\
\hline Efeito hepatoprotetor & $1(1,4)$ \\
\hline Efeito sobre as colinesterases - DM & $1(1,4)$ \\
\hline Efeito cicatrizante & $1(1,4)$ \\
\hline Envelhecimento & $1(1,4)$ \\
\hline Efeito anti-hipertensivo & $1(1,4)$ \\
\hline Efeito anti-helmíntico - Ancylostoma & $1(1,4)$ \\
\hline Remodelação cardíaca pós infarto & $1(1,4)$ \\
\hline Inibição de células tumorais & $1(1,4)$ \\
\hline
\end{tabular}

Nota: algumas produções avaliaram mais de uma ação do Alecrim ou de seus compostos. Fonte: Autores.

Algumas das pesquisas realizadas (15,5\%) envolveram o desenvolvimento de produtos, entre eles, dentífrico $(2,8 \%)$, nanoemulsão direcionada ao tratamento de herpes $(2,8 \%)$, enxaguatório bucal $(1,4 \%)$, fitocosmético clareador $(1,4 \%)$, gelcreme $(1,4 \%)$, emulsão cicatrizante $(1,4 \%)$, formulação cosmética fotoprotetora $(1,4 \%)$, nanoemulsão anti-inflamatória $(1,4 \%)$ e nanocápsula combinando óleo essencial com medicações convencionais (adjuvante) para potencializar efeito.

\section{Discussão}

Os resultados desta investigação demonstram tendência crescente nas pesquisas com o Alecrim ou de seus compostos com potencial terapêutico à saúde, isto é, há um aumento quantitativo no decorrer dos anos. Estas investigações foram desenvolvidas por diversas universidades do Brasil, localizadas principalmente na região Sul e Sudeste do país e destaque para a Universidade Federal de Pelotas e Universidade Federal de Santa Maria com maior número de produções e para os 
programas de pós-graduação em Ciências Farmacêuticas, Veterinária e ciências dos alimentos.

$\mathrm{O}$ aumento nas pesquisas com plantas medicinais e fitoterápicos no Brasil, incluindo o Alecrim, pode ser esclarecida pela crescente insatisfação da população em relação aos medicamentos sintéticos, efeitos adversos, alto custo, dificuldade de acesso, demanda por alternativas terapêuticas e naturais para tratamento de doenças e também pela valorização da medicina tradicional, estímulo a sua utilização e pesquisa desde o desenvolvimento do SUS, da implantação da Política Nacional de Plantas Medicinais e Fitoterápicos em 2006, do Programa Nacional de Plantas Medicinais e Fitoterápicos, em 2008 (Bitencourt \& Melo, 2016).

No âmbito da pós-graduação, justifica-se o maior número de pesquisas realizadas nas regiões Sul e Sudeste por estas concentrarem o maior número de programas de pós-graduação no Brasil. O envolvimento dos programas de pós-graduação em Ciências Farmacêuticas nas pesquisas com Alecrim e de seus compostos reforça a competência do farmacêutico em assuntos pertinentes às atividades de produção e utilização terapêutica das plantas medicinais, seus derivados e fitoterápicos, na promoção do acesso com qualidade, segurança e eficácia e na participação das pesquisas visando o desenvolvimento de novos fitoterápicos, ou de novas indicações/posologias para os já registrados (Resolução CFF no 477, 2008). Em relação aos programas de pós-graduação em Veterinária, o desenvolvimento de pesquisas com plantas medicinais e fitoterápicos tem sido motivado pelo fácil acesso e baixo custo a esses produtos, aliado a necessidade de comprovação de sua eficácia terapêutica, toxicidade, frequência de uso e concentração adequada para animais (Batista et al., 2017). Em se tratando das investigações na área das Ciências dos Alimentos, destaca-se que os produtos naturais têm ganhado destaque como tecnologia emergente para a conservação de alimentos como substitutos de compostos artificiais, que auxiliam no tempo de prateleira, mantêm o sabor e o aroma, com importância mundial para a indústria alimentícia e também com aplicação na medicina tradicional, atendendo as exigências dos consumidores (Pereira, 2017). Constata-se ausência de produções no âmbito dos programas de Pós-Graduação em Enfermagem sobre o tema.

A quantidade variada de universidades que tiveram uma ou duas produções sobre o tema e o destaque da Universidade Federal de Pelotas com cinco publicações no mesmo programa e da Universidade Federal de Santa Maria com quatro produções em dois programas, evidenciam o panorama atual da construção do conhecimento, no qual há uma transição entre o desenvolvimento de pesquisas isoladas, em busca de um maior quantitativo de publicações, para pesquisas de maior qualidade construídas de forma colaborativa e em grupos de pesquisa, em torno de linhas comuns, com maior impacto social e econômico (Moreira et al., 2015).

Os resultados evidenciam também que pouco mais da metade das pesquisas com o Alecrim e/ou seus componentes foram realizadas in vitro, em especial porque avaliaram o efeito sobre microorganismos, citotoxicidade e genotoxicidade. Em relação aos estudos in vivo, a maioria absoluta envolveu ratos ou camundongos e duas foram com humanos (estudos que avaliaram dentífricos). Cabe salientar que, embora, na atualidade se busque a utilização de métodos substitutivos aos modelos animais, nem sempre isso é possível, devido à complexidade das interações entre células, tecidos e órgãos que ocorrem nos organismos vivos. Ademais, testes com novos produtos/fármacos auxiliam na atenuação ou prevenção de seus efeitos colaterais em humanos a partir de observações prévias in vivo (Guimarães et al., 2016).

No que diz respeito aos achados das produções analisadas, mais da metade avaliou a ação antimicrobiana e antibiofilme do Alecrim ou de seus compostos. Verificou-se que o óleo essencial de Alecrim inibiu cepas de bactérias Grampositivas como Staphylococcus aureus (Wanderley, 2015; Pereira, 2017; Luchesi, 2017), Klebsiella pneumoniae (Wanderley, 2015), Bacillus cereus (Pereira, 2017) e Pseudomonas spp (Luchesi, 2017; Guterres, 2019, 2015), assim como inibiu bactérias Gram-negativas, Escherichia coli (Pereira, 2017; Ramos, 2014) e Salmonella entérica (Pereira, 2017; Luchesi, 2017).

Pesquisa não encontrou atividade inibitória do óleo essencial de Alecrim e de sua fração majoritária cineol sobre Staphylococcus spp (Dal Pozzo, 2010). Nesse sentido, a utilização de nanocápsulas com óleo essencial de Alecrim associado à 
Mupirocina melhorou a atividade de ambos contra esta bactéria (Rubenick, 2017). Também, evidenciou-se que o óleo essencial de Alecrim pode atuar como modificador da atividade antibiótica ao reduzir a Concentração Inibitória Mínima destes medicamentos, entre os quais tetraciclina, eritromicina e norfloxacina (Cirino, 2014).

Quanto à atividade antifúngica, os achados são discordantes sobre o efeito do óleo essencial. Estudos indicaram atividade antifúngica em dermatófitos, Pythium insidiosum (Fonseca, 2011), Microsporum canis e Trichophyton rubrum (Silva, 2018a), porém uma pesquisa não encontrou efeito em dermatomicoses (Mateus, 2016). Em relação às espécies de Candida, duas investigações não encontraram atividade inibitória (Ludwig, 2018; Pozzati, 2007), enquanto que outra pesquisa evidenciou atividade inibitória e fungicida em isolados de Candida de portadores de próteses removíveis (Gauch, 2014). Em relação aos vírus, nanoemulsão contendo óleo essencial apresentou inibição contra os vírus da herpes, maior especificidade pelo sorotipo 2 (Cavalcanti, 2017; Zibetti, 2016). Cabe esclarecer aqui que os óleos essenciais são produtos aromáticos altamente voláteis obtidos de plantas, principalmente no estado líquido, em geral, são considerados metabólitos secundários e utilizados pelas plantas para a fertilização e proteção contra patógenos (Naeem et al., 2018). O processo de extração influencia diretamente na composição química dos óleos e consequentemente a atividade antimicrobiana, assim como o sinergismo de seus compostos (Pereira, 2010) e a variação sazonal durante o desenvolvimento da planta (Lemos, 2014).

Estudo que utilizou extrato bruto de Alecrim indicou boa atividade microbiana, inclusive contra Staphylococcus aureus resistente à meticilina (MRSA) e Klebsiella pneumoniae produtora de carbepenemase (KPC) (Dalmarco, 2012). Observou-se também atividade do extrato contra biofilmes (Oliveira, 2016). Outra investigação demonstrou que o Rosmarinus officinalis possui atividade frente a patógenos Gram positivos, Staphylococcus aureus e Bacillus subtilis e que atua sinergicamente com o antifúngico nistatina (Costa, 2013). Pesquisa com os constituintes ácido ursólico, ácido úsnico e a combinação entre o ácido difractáico e o ácido úsnico evidenciou que todos estes são eficazes frente a microrganismos resistentes (Tozatti, 2016).

Extrato padronizado de ácido rosmarínico também apresentou atividade inibitória frente às bactérias e leveduras (Chaul, 2015; Degani Filho, 2018). O extrato hidroalcoólico puro de Alecrim e diluído até 1/16 apresentou ação antimicrobiana sobre cepas de Streptococcus sanguinis, Streptococcus mutans, Streptococcus sobrinus e Lactobacillus casei (Silva, 2005). Extrato etanólico, ainda, mostrou-se eficiente para o controle de Shigella Flexneri - bactéria causadora de infecção intestinal (Alvarenga, 2004). Extrato aquoso promoveu aceleração da resolução de otite externa infecciosa, em ratos, por Staphylococcus aureus (Fontoura, 2014). Tanto os extratos aquosos como os hidroalcóolicos apresentaram baixa atividade contra Sporothrix spp e Candida (Waller, 2015; Mardegan, 2007). Contudo, o extrato metanólico apresentou atividade com alta sensibilidade das leveduras (Anibal, 2007).

Ainda, em relação a atividade antimicrobiana, enxaguatório bucal contendo extrato etanólico apresentou efeito contra biofilme de Streptococcus Mutans e Candida Albicans (Yamamoto, 2013). O extrato aquoso produziu inibição da enzima Glicosiltransferase de Streptococcus Mutans com consequente inibição de substâncias envolvidas na cárie (Battagin, 2010). Dentifrício com extrato de Alecrim, testado em humanos e in vitro, apresentou capacidade de inibir o crescimento das bactérias supramencionadas e também de Staphylococcus aureus e Lactobacillus casei (Valones, 2008, 2016; Tenorio, 2014) e se mostrou efetivo quanto ao sangramento gengival e na redução de placa bacteriana em comparação ao dentifrício convencional (Valones, 2016; Tenorio, 2014). Explicita-se que os extratos são preparações líquidas, semissólidas ou sólidas, obtidas a partir de plantas inteiras ou de suas partes com utilização de métodos de extração e solventes (ANVISA, 2021). Os dados permitem inferir que o tipo de substância proveniente do Alecrim, seja ela em forma de óleo essencial, extrato bruto, extrato alcoólico, extrato aquoso e outros, pode apresentar efeitos diferentes sobre os microrganismos, bem como sua concentração e o sinergismo entre os componentes.

Em relação ao efeito anti-inflamatório, os estudos evidenciaram que o extrato bruto, frações, óleo essencial e 
compostos isolados de Alecrim possuem este tipo de efeito (Oliveira, 2016; Benicá, 2010; Silva, 2008; Mueller, 2011; Cavalcanti, 2017; Warken, 2016; Rosa, 2013; Santos, 2013; Takaki, 2006; Borges, 2018) em especial pela inibição da produção de citocinas próinflamatórias (Oliveira, 2016), redução do influxo de leucócitos e da exsudação (Rosa, 2013). Verifica-se essa atividade, no caso de óleos essenciais e do ácido rosmarínico, em processos agudos e crônicos, e ainda efeito antinociceptivo (Santos, 2013; Faria, 2005), porém, o óleo mostrou-se irritante ao epitélio auditivo em ratos (Mueller, 2011). Estudos com emulsão ou nanoemulsão, para além do efeito anti-inflamatório, demonstraram que estas auxiliam na regeneração tecidual, no processo de cicatrização e tornam os compostos mais disponíveis nos locais alvo (Cavalcanti, 2017; Warken, 2016).

Quanto ao efeito antioxidante, constatou-se que, tanto o extrato aquoso e frações fenólicas, quanto extrato etanólico, produzem esta ação (Afonso, 2010; Silva, 2012; Gonçalves, 2017; Silva, 2019; Zamberlan, 2018), que pode ser observada por redução na produção de espécies reativas em condições basais, hiperglicêmicas (Silva, 2012) e na artrite (Gonçalves, 2017), elevação da atividade das enzimas antioxidantes no fígado e cérebro (SILVA, 2008), aumento da longevidade e da resistência ao estresse (Zamberlan, 2018). Fitocosmético contendo extrato padronizado em ácido rosmarínico, além de efeito antioxidante, produziu também ação clareadora e pode ser uma alternativa a este tipo de substância existente no mercado (Silva, 2019).

Outra pesquisa com formulação contendo extrato aquoso e/ou extrato etanólico demonstrou que sua utilização pode ser fonte tópica de compostos antioxidantes com diminuição dos danos oxidativos da pele e efeito fotoquimiopreventivo (Takayama, 2018). Ação fotoprotetora também foi verificada em pesquisa com nanoformulação contendo óleo essencial, que auxiliou na preservação da cor e prevenção de danos em fibra capilar irradiada por ultravioleta (Desmarais, 2018).

Nota-se que o efeito anti-inflamatório e antioxidante do Alecrim são responsáveis pelo seu potencial terapêutico para doenças crônicas como a artrite e a diabetes (Gonçalves, 2017; Dutra, 2014; Silva, 2008), no tratamento da dor, na promoção da cicatrização tecidual e também para o desenvolvimento de fitocosméticos. Estudos reforçaram o efeito do extrato aquoso e fração fenólica do Alecrim em algumas condições crônicas indicando redução nos níveis séricos de colesterol total, lipoproteína de baixa densidade - LDL -c (Afonso, 2010) e nos níveis de hemoglobina glicosilada (Silva, 2008).

As produções analisadas, igualmente, indicaram efeito antidepressivo promovido pelo extrato hidroalcoólico de Rosmarinus officinalis, dos compostos carnosol, ácido betulínico e ácido ursólico (Machado, 2012; Hryb, 2017), pelo extrato bruto e óleo essencial (Dalmarco, 2012). Verificou-se que o extrato hidroalcoólico pode reverter as alterações comportamentais - hiperatividade e comportamento anedônico (Machado, 2012), que o óleo essencial possui atividade sobre transtorno obsessivo compulsivo - TOC (Lima, 2010) e ansiedade (Arruda, 2014). Contatou-se que o ácido rosmarínico apresenta efeito neuroprotetor frente à corticosterona secretada diante de estresse pós-traumático (Aguiar, 2019) e devido à prevenção do início da Doença de Parkinson ao elevar o conteúdo de dopamina e serotonina (Silva, 2018b). Infere-se assim, que o Alecrim pode ser utilizado como auxiliar no tratamento de depressão, ansiedade, TOC e doenças degenerativas como o Parkinson.

Algumas publicações analisaram a citotoxicidade e genotoxicidade e constataram que o extrato vegetal de Alecrim não é mutagênico e não alterou a viabilidade das linhagens celulares (macrófagos de camundongo, fibroblastos gengivais humanos, linhagem tumoral de carcinoma mamário e de carcinoma cervical) (Oliveira, 2016), bem como o extrato aquoso, que manteve a viabilidade de hepatócitos humanos (Silva, 2012). No entanto, observou-se que o óleo essencial apresentou citotoxicidade quando avaliado efeito em melanoma (Silva, 2016), em células renais (Guterres, 2019), exceto em felinos (Kubiça, 2012), e espermáticas (Guterres, 2019) e foi observado que pode destruir hemácias causando a liberação da hemoglobina (Rezende, 2016). O óleo, assim como o 1,8 cineol, também deve ser evitado em gestantes porque interfere no desenvolvimento fetal (Santos, 2017). Diante destes resultados, recomenda-se mais pesquisas sobre o efeito do Alecrim e seus compostos em diferentes formas de apresentação que avaliem a citotoxicidade.

Ainda em relação aos achados das produções analisadas, verificaram-se: redução da motilidade gástrica por ação dos 
extratos aquoso e etanólico de Alecrim (Carnevali, 2012); prevenção de lesões gastrointestinais como efeito do extrato etanólico (Amaral, 2016) e redução do índice de lesão ulcerativa e área de lesão gástrica como efeito do ácido rosmarínico (Nascimento, 2016); atividade hepatoprotetora por pré-tratamento com óleo essencial de Alecrim (Pinho, 2013); atividade antihipertensiva do óleo essencial (Neves, 2019); atenuação da remodelação cardíaca após o infarto do miocárdio em ratos suplementados com folhas secas de Alecrim (Rafacho, 2015); inibição da eclodibilidade de ovos de Ancylostoma spp por ação de óleo essencial (Azambuja, 2015); e ausência de atividade do óleo essencial contra Trypanossoma Cruzi (Azeredo, 2013). Diante do exposto, observam-se que há investigações avaliando o efeito de substâncias provenientes do Alecrim e/ou seus compostos em diferentes doenças, microorganismos e parasitas, com resultado positivo ou não em benefício da saúde humana.

Algumas pesquisas envolveram o desenvolvimento de produtos com substâncias derivadas de Alecrim, entre eles, dentífrico, nanoemulsão direcionada ao tratamento de herpes, enxaguatório bucal, fitocosmético clareador, gel-creme, emulsão cicatrizante, formulação cosmética fotoprotetora, nanoemulsão anti-inflamatória e nanocápsula combinando óleo essencial com medicações convencionais (adjuvante) para potencializar efeito. Embora os estudos com o desenvolvimento de produtos a base de plantas corresponderam a um pequeno número, salienta-se que estudo bibliométrico evidenciou que as pesquisas mundiais sobre plantas medicinais como fontes de produtos para a saúde estão em expansão, em especial com o desenvolvimento de novos medicamentos ou compostos ativos e apontou o Brasil como um dos grandes pesquisadores na área, particularmente com investigações sobre a segurança e a eficácia desses produtos (Salmerón-Manzano et al., 2020). Assim, a presente pesquisa permitiu identificar tendência crescente nas produções científicas sobre o potencial terapêutico do Alecrim à saúde ao mesmo tempo em que se verificam lacunas no conhecimento.

Como limitação deste manuscrito, salienta-se a dificuldade de incluir as produções de 2019 em virtude da indisponibilidade online e a falta da avaliação das produções sobre o potencial terapêutico do Alecrim à saúde em programas de pós-graduação internacionais, o que de certa forma abre portas a uma nova pesquisa.

\section{Considerações Finais}

Esta pesquisa apontou como tendência das produções científicas brasileiras acerca do potencial terapêutico do Alecrim à saúde, o crescimento das pesquisas sobre o tema, o envolvimento de diversas universidades do Brasil, com destaque para a Universidade Federal de Pelotas e Universidade Federal de Santa Maria e para os programas de pós-graduação em Ciências Farmacêuticas, Veterinária e ciências dos alimentos. Demonstrou ausência de estudos provenientes de Programas de Pós-Graduação em Enfermagem e abre portas para a inserção destes nas pesquisas sobre a temática, de forma a contribuir com a construção do conhecimento e aplicação prática da fitoterapia. Indicou prevalência de pesquisas isoladas, mas transição para aquelas construídas de forma colaborativa em torno de linhas comuns. Demonstrou inclinação para estudos in vitro, seguidos dos in vivo, em especial ratos e camundongos e carência de estudos em humanos.

A maior parte das pesquisas tendeu avaliar a ação antimicrobiana e antibiofilme do Alecrim e seus compostos (incluindo extratos e óleos), seguido do efeito anti-inflamatório, antioxidante, antidepressivo e citotoxicidade, evidenciando potencial terapêutico contra microrganismos, inclusive resistentes, tratamento complementar de doenças crônicas não transmissíveis como artrite, diabetes, hipertensão, Parkinson, depressão e outras. Algumas produções científicas também envolveram desenvolvimento de produtos acompanhando a tendência mundial das pesquisas com plantas medicinais. Ainda, permitiu identificar a necessidade de aprofundamento e do desenvolvimento de pesquisas futuras que abordem o efeito do Alecrim e seus compostos em microrganismos resistentes; na prevenção e tratamento complementar de doenças crônicas como hipertensão e diabetes; comparação da efetividade terapêutica dos diferentes extratos e suas composições; que avaliem o potencial desses produtos como adjuvantes ao tratamento convencional e sua influência na eficácia desse. 


\section{Agradecimentos}

Os autores agradecem ao Conselho Nacional de Desenvolvimento Científico e Tecnológico (CNPq) e à Fundação de Amparo à Pesquisa do Rio Grande do Sul (FAPERGS) pelas bolsas de iniciação científica.

\section{Referências}

Agência Nacional de Vigilância Sanitária (2021). Formulário de Fitoterápicos da Farmacopeia Brasileira (2nd ed.). Farmacopeia Brasileira. https://www.gov.br/anvisa/pt-br/assuntos/farmacopeia/formulario-fitoterapico/arquivos/2021-fffb2-final-c-capa2.pdf

Afonso, M.S. (2010). Avaliação do efeito do extrato aquoso e da fração fenólica livre do Alecrim (Rosmarinus officinalis L.) sobre o estado antioxidante e o perfil lipídico em ratos com hipercolesterolemia induzida pela dieta [Dissertação de Mestrado, Universidade de São Paulo]. Biblioteca Digital USP. https://doi.org/10.11606/D.9.2011.tde-17012011-163651

Aguiar, R. M. (2019). Avaliação do efeito neuroprotetor do ácido rosmarínico em um modelo pré-clínico de transtorno de estresse pós-traumático em camundongos [Dissertação de Mestrado, Universidade Federal do Espírito Santo]. Portal UFES. http://portais4.ufes.br/posgrad/te ses/tese_13303_Disserta\%E7\%E3o\%20Final\%20-\%20Rafael\%20Moraes\%20Aguiar.pdf

Alvarenga, A. L. (2004). Efeito antibacteriano in vitro de extratos vegetais sobre bactérias patogênicas [Dissertação de Mestrado, Universidade Federal de Lavras. Repositório UFLA. http://repositorio.ufla.br/jspui/bitstream/1/36140/1/DISSERTA\%C3\%87\%C3\%830_Efeito\%20antibacteriano\%20in\%20vitro\% 20de\%20extratos\%20vegetais\%20sobre\%20bact\%C3\%A9rias\%20patog\%C3\%AAnicas.pdf

Amaral, G. P. (2016). Propriedades de proteção gastrointestinal da Rosmarinus officinalis l. em associação a testes microbiológicos e antioxidantes in vitro e ex vivo [Tese de Doutorado, Universidade Federal de Santa Maria]. Repositório Digital da UFSM. https://repositorio.ufsm.br/handle/1/18218

Andrade, J. M., Faustino, C., Garcia, C., Ladeiras, C. P. R \& Rijo, P. (2018). Rosmarinus officinalis L.: an update review of its phytochemistry and biological activity. Future Science, 4(4), FSO283. https://doi.org/10.4155/fsoa-2017-0124.

Anibal, P. C. (2007). Potencial de ação antimicrobiana in vitro de extratos de plantas na inibição de Candida spp, Streptococcus mutans e Staphylococcus aureus [Dissertação de Mestrado, Universidade Estadual de Campinas]. Repositório da Produção Científica e Intelectual da Unicamp. http://repositorio.unicamp.br/jspui/handle/REPOSIP/289352

Arruda, T. V. (2014). Efeitos dos óleos essenciais de Alecrim (Rosmarinus officinalis) e Petitgrain (Citrus aurantium L.) em modelos comportamentais de atividade motora, depressão, ansiedade e aprendizado em ratos [Dissertação de Mestrado, Universidade Metodista de São Paulo]. Biblioteca Digital de Teses e Dissertações Metodista. http://tede.metodista.br/jspui/handle/tede/1358

Azambuja, R. H. M. (2015). Prospecção dos óleos essenciais da família Lamiaceae, sobre ovos de Ancylostoma spp. [Dissertação de Mestrado, Universidade Federal de Pelotas]. Portal Institucional UFPel. https://institucional.ufpel.edu.br/projetos/id/p5088

Azeredo, C. M. O. (2013). Efeito inibitório de óleos essenciais sobre Trypanosoma Cruzi [Dissertação de Mestrado, Universidade Federal do Paraná]. Acervo digital UFPR. https://acervodigital.ufpr.br/bitstream/handle/1884/35243/R\%20-\%20D\%20\%20CAMILA\%20MARIA\%20OLIVEIRA\%20DE\%20 AZEREDO.pdf?sequence=1\&isAllowed=y

Batista, F. T., Lacerda, G. D., Silva, J. R. S., Rodrigues, L. P. T., Feitosa, T. P. \& Mustafa, V. S. (2017). O uso de plantas medicinais na medicina veterinária riscos e benefícios. Revista Científica do Curso de Medicina Veterinária, 4(2), 62-74. http://revista.faciplac.edu.br/index.php/Revet/article/view/337

Battagin, J. (2010). Cinética enzimática e efeito de extratos naturais na atividade da enzima glicosiltransferase de Streptococcus mutans [Dissertação de Mestrado, Universidade São Francisco]. https://www.livrosgratis.com.br/ler-livro-online-95970/cinetica-enzimatica-e-efeito-de-extratos-naturais-na-atividadeda-enzima-glicosiltransferase-de-streptococcus-mutans

Benicá, J. P. (2010). Estudo da eficácia anti-inflamatória da Rosmarinus officinalis L., em camundongos, utilizando-se o modelo da pleurisia induzida pela carragenina [Dissertação de Mestrado, Universidade Federal de Santa Catarina]. Repositório Institucional UFSC. https://repositorio.ufsc.br/handle/123456789/94427

Bitencourt, L. C. \& Melo, M. B. (2016). A utilização de plantas medicinais e fitoterápicos na rede de atenção básica de saúde no município de Aracaju-SE. Ciência Biológicas e de Saúde, 3(3), 165-176. https://periodicos.set.edu.br/cadernobiologicas/ article/view/2974

Borges, F. V. \& Sales, M. D. C. (2018). Políticas públicas de plantas medicinais e fitoterápicos no Brasil: sua história no sistema de saúde. Pensar Acadêmico, 16(1), 13-27. https://doi.org/10.21576/rpa.2018v16i1.18

Borges, R. S. (2018). Estudo da atividade anti-inflamatória de nanoemulsões a base do óleo essencial de Rosmarinus officinalis L. [Tese de Doutorado, Universidade Federal do Amapá]. UniFAp. https://www2.unifap.br/ppgdif/files/2017/10/TESE_RAPHAELLE_SOUSA_BORGES_08-06-2018.pdf

Carnevali, T. R. (2012). Avaliação radiológica da motilidade gástrica de ratos wistar, submetidos à ação da eletroacupuntura e de extratos de Rosmarinus officinalis [Dissertação de Mestrado, Universidade Federal de Pelotas]. Guaiaca UFPel. http://guaiaca.ufpel.edu.br/handle/123456789/2476

Cavalcanti, J. F. (2017). Avaliação da atividade inibitória dos óleos essenciais de Rosmarinus officinalis e Illicium verum e suas respectivas nanoemulsões sobre os alfaherpesvírus humanos 1 e 2 e o mastadenovírus humano 19 [Tese de Doutorado, Universidade Federal do Rio de Janeiro]. Plataforma Sucupira. https://sucupira.capes.gov.br/sucupira/public/consultas/coleta/trabalhoConclusao/viewTrabalhoConclusao.jsf?popup=true\&id_trabalho=5033629

Chaul, L. T. (2015). Caracterização e Determinação da Atividade Antimicrobiana dos Extratos Brutos e Frações de Rosmarinus officinalis Linn.(alecrim), Origanum vulgare Linn. (orégano) e Capsicum chinense Jacq. (pimenta habanero) [Dissertação de Mestrado, Universidade Federal de Goiás,]. 2015. Biblioteca Digital de Teses e Dissertações UFG. https://repositorio.bc.ufg.br/tede/handle/tede/4560 
Cirino, I. C. S. (2014). Modulação da resistência a drogas por óleos essenciais em linhagens de Staphylococcus aureus [Dissertação de Mestrado, Universidade Federal da Paraíba]. Repositório Institucional UFPB. https://repositorio.ufpb.br/jspui/bitstream/tede/3665/1/arquivototal.pdf

Resolução CFF n 477 de 28 de maio de 2008. Dispõe sobre as atribuições do farmacêutico no âmbito das plantas medicinais e fitoterápicos e dá outras providências. Conselho Federal De Farmácia. https://www.legisweb.com.br/legislacao/?id=108740

Costa, G. M. (2013). Avaliação da atividade antimicrobiana de plantas medicinais [Dissertação de Mestrado, Universidade Estadual de Maringá]. Plataforma Sucupira. https://sucupira.capes.gov.br/sucupira/public/consultas/coleta/trabalhoConclusao/viewTrabalhoConclusao.jsf?popup=true\&id_trabalho=605193

Dal Pozzo, M. (2010) Mastite bovina e caprina: suscetibilidade de isolados de Staphylococcus spp. frente aos óleos essenciais extraídos de condimentos [Dissertação de Mestrado, Universidade Federal de Santa Maria]. Repositório Digital da UFSM. https://repositorio.ufsm.br/handle/1/10758

Dalmarco, J. B. (2012). Estudo das propriedades químicas e biológicas de Rosmarinus Officinalis L. [Tese de Doutorado, Universidade Federal de Santa Catarina]. Repositório Institucional UFSC. https://repositorio.ufsc.br/xmlui/handle/123456789/100930

Degani Filho, G. P. (2018). Avaliação da eficácia conservante e da estabilidade de géis-creme contendo extrato padronizado em ácido rosmarínico de folhas de Rosmarinus officinalis L. [Dissertação de Mestrado, Universidade Federal de Goiás]. Plataforma Sucupira. https://sucupira.capes.gov.br/sucupira/public/consultas/coleta/trabalhoConclusao/viewTrabalho Conclusao.jsf?popup=true\&id_trabalho=8286173

Desmarais, G. C. (2018). Desenvolvimento de formulações cosméticas nanoestruturadas contendo o óleo essencial de Rosmarinus officinalis L. e avaliação do efeito na fibra capilar antes e após exposição à radiação UV [Dissertação de Mestrado, Universidade Federal Fluminense]. Repositório Institucional da Universidade Federal Fluminense. https://app.uff.br/riuff/handle/1/8651

Dutra, E. J. M. (2014). Avaliação do efeito de extratos de batata e Alecrim sobre as colinesterases em um modelo de diabetes tipo 1 [Dissertação de Mestrado, Universidade Federal de Santa Maria]. Repositório Digital da UFSM. https://repositorio.ufsm.br/handle/1/11250

Faria, L. R. D. (2005). Validação farmacológica do óleo essencial de Rosmarinus officinalis L. (Alecrim): atividades antiinflamatória e analgésica [Dissertação de Mestrado, Universidade Jose do Rosario Vellano]. Biblioteca Digital de Teses e Dissertações UNIFENAS. http://tede2.unifenas.br:8080/jspui/handle/jspui/134

Fonseca, A. O. da S. (2011). Ação antifúngica dos óleos essenciais de Origanum vulgare e Rosmarinus officinalis frente a isolados de Pythium insidiosum e Dermatófitos [Dissertação de Mestrado, Universidade Federal de Pelotas]. Guaiaca UFPel. http://guaiaca.ufpel.edu.br/handle/123456789/2570

Fontoura, E. G. (2014). Rosmarinus officinalis L. e Triticum aestivum no tratamento da otite externa infecciosa [Dissertação de Mestrado, Universidade Federal de Pelotas]. Guaiaca UFPel. http://guaiaca.ufpel.edu.br/handle/123456789/2511

Gauch, L. M. R. (2014). Avaliação da atividade antifúngica do óleo essencial de Alecrim (Rosmarinus officinalis) sobre leveduras do gênero Candida recuperadas da cavidade oral de portadores de prótese removível [Tese de Doutorado, Universidade Federal do Pará]. Plataforma Sucupira. https://sucupira.capes.gov.br/sucupira/public/consultas/coleta/trabalho Conclusao/viewTrabalhoConclusao.jsf?popup=true\&id_trabalho=720260

Gonçalves, G. A. (2017). Extratos hidroalcoolicos de bagaço de uva Merlot e compostos hidrossolúveis de Alecrim melhoram os estados oxidativo e inflamatório de ratos com artrite induzida por adjuvante [Tese de Doutorado, Universidade Estadual de Maringá]. Plataforma Sucupira. https://sucupira.capes.gov.br/sucupira/public/consultas/coleta/trabalhoConclusao/view TrabalhoConclusao.jsf? popup=true\&id_trabalho=5111569

Guimarães, M. V., Freire, J. E. C. \& Menezes, L. M. B. (2016). Utilização de animais em pesquisas: breve revisão da legislação no Brasil. Revista Bioética, 24(2),217-224. http://dx.doi.org/10.1590/1983-80422016242121

Guterres, K. A. (2015). Microrganismos de lesões cutâneas de pequenos animais: Resistência a antimicrobianos e bioprospecção de extratos de plantas da família Lamiaceae e Fabaceae [Dissertação de Mestrado, Universidade Federal de Pelotas]. Guaiaca UFPel. http://guaiaca.ufpel.edu.br/handle/prefix/3417

Guterres, K. A. (2019). Prospecção e toxicidade de óleos essenciais de plantas da família Lamiaceae em Pseudomonas spp. isoladas de cães e perfil de resistência da bactéria [Tese de Doutorado, Universidade Federal de Pelotas]. Plataforma Sucupira. https://sucupira.capes.gov.br/sucupira/p ublic/consultas/coleta/trabalhoConclusao/viewTrabalhoConclusao.jsf? popup=true\&id_trabalho=7614764

Hryb, A. B. R. (2017). Estudo dos mecanismos envolvidos no efeito tipo - antidepressivo e neuroprotetor do ácido ursólico [Tese de Doutorado, Universidade Federal de Santa Catarina]. Repositório Institucional UFSC. https://repositorio.ufsc.br/handle/123456789/183618

Kubiça, T. F. (2012). Atividade antiviral de óleos essenciais e monoterpenos contra vírus de bovinos e felinos como potenciais modelos para vírus humanos [Dissertação de Mestrado, Universidade Federal de Santa Maria]. Repositório Digital UFSM. https://repositorio.ufsm.br/handle/1/5960?show=full

Lemos, M. F. (2014). Efeitos da variação sazonal sobre a composição e atividade biológica de óleos essenciais [Dissertação de Mestrado, Universidade de Vila Velha]. Repositório UVV. https://repositorio.uvv.br/handle/123456789/220?locale=es

Lima, V. M. (2010). Avaliação da atividade antidepressiva e ansiolítica do óleo essencial de Rosmarinus officinalis L. [Dissertação de Mestrado, Universidade Estadual Paulista]. Repositório Institucional UNESP. https://repositorio.unesp.br/handle/11449/91650\#: :text=Os\%20resultados\%20s $\%$ C3\%A3o\%20promissores $\% 2 \mathrm{C} \% 20$ pois,no\%20modelo\%20de\%20ansiedade\%20generalizada.

Luchesi, L. A. (2017). Atividade antibacteriana, antifúngica e antioxidante de óleos essenciais [Dissertação de Mestrado, Universidade Tecnológica Federal do Paraná]. Repositório Institucional da UTFPR. https://repositorio.utfpr.edu.br/jspui/handle/1/2678

Ludwig, A. (2018). Suscetibilidade in vitro de Candida rugosa frente à antifúngico e óleos essenciais [Dissertação de Mestrado, Universidade Federal de Santa Maria]. Repositório Digital da UFSM. https://repositorio.ufsm.br/handle/1/20424

Machado, D. G. (2012). Investigação do potencial antidepressivo de Rosmarinus officinalis [Tese de Doutorado, Universidade Federal de Santa Catarina]. Repositório Institucional UFSC. https://repositorio.ufsc.br/handle/123456789/96131 
Mardegan, R. C. (2007). Atividade Inibitória de Extratos Vegetais sobre Candida spp e sobre Proteinases sintetizadas por Candida albicans [Tese de Doutorado, Universidade Estadual de Campinas]. Repositório da Produção Científica e Intelectual da Unicamp. http://repositorio.unicamp.br/jspui/handle/REPOSIP/289371

Mateus, W. S. (2016). Avaliação dos efeitos fungistáticos e fungicidas de óleos essenciais em microrganismos causadores de dermatomicoses [Dissertação de Mestrado, Universidade Federal do Triângulo Mineiro]. Biblioteca Digital de Teses e Dissertações Universidade Federal do Triângulo Mineiro. http://bdtd.uftm.edu.br/handle/tede/362

Mendes, D. S., Moraes, F. S. de, Lima, G. de O., Silva, P. R. da, Cunha, T. A., Crossetti, M da G. O. \& Riegel, F. (2019). Benefícios das práticas integrativas e complementares no cuidado de enfermagem. Journal Health NPEPS, 4(1), 302-318. http://dx.doi.org/10.30681/252610103452

Moreira, J. R., Vilan Filho, J. L. \& Mueller, S. P. P. (2015). Características e produção científica dos grupos de pesquisa do CNPq/DGP nas áreas de Ciência da Informação e Museologia (1992 - 2012). Perspectivas em Ciências da Informação, 20(4), 93-106. http://dx.doi.org/10.1590/1981-5344/2460

Mueller, E. N. (2011). Microclima do canal auditivo de cães e efeito do Rosmarinus officinalis L. e do Triticum vulgare no tratamento da otite externa experimental [Tese de Doutorado, Universidade Federal de Pelotas]. Repositório Institucional da UFPel. http://guaiaca.ufpel.edu.br/handle/123456789/2578

Naeem, A., Abaas, T., Ali, T. M. \& Hasnain, A (2018). Essential Oils: Brief Background and Uses. Annals of Short Report, 1(1), 1016. http://www.remedypublications.com/open-access/essential-oils-brief-background-and-uses-516.pdf

Nascimento, R. F. (2016). Avaliação da atividade gastroprotetora do ácido rosmarínico em modelos animais [Dissertação de Mestrado, Universidade Federal da Paraíba]. Repositório Institucional da UFPB. https://repositorio.ufpb.br/jspui/handle/tede/9480?locale=pt_BR

Nematolahi, P., Mehrabani, M., Karami-Mohajeri, S. \& Dabaghzadeh, F. (2018). Effects of Rosmarinus officinalis L. on memory performance, anxiety, depression, and sleep quality in university students: A randomized clinical trial. Complementary Therapies in Clinical Practices, 30, $24-28$. https://linkinghub.elsevier.com/retrieve/pii/S1744388117304723

Neves, J. A. (2019). Atividade do óleo essencial de Rosmarinus Officinalis L. Livre e complexado em $\beta$-ciclodextrina em ratos hipertensos - l-name [Tese de Doutorado, Fundação Universidade Federal do Piauí]. Repositório Institucional da UFPI. https://repositorio.ufpi.br/xmlui/handle/123456789/2022

Oliveira, J. R. (2016). Avaliação de atividades biológicas dos extratos de Rosmarinus officinalis L. (Alecrim) e Thymus vulgaris L. (tomilhon) [Tese de Doutorado, Universidade Estadual de São Paulo]. Repositório Institucional UNESP. https://repositorio.unesp.br/handle/11449/137865

Oliveira, J. R., Camargo, S. E. A. \& Oliveira, L. D. (2019). Rosmarinus officinalis L. (rosemary) as therapeutic and prophylactic agent. Journal of Biomedical Science, 26(1),5. http://dx.doi.org/10.1186/s12929-019-0499-8

Organização Mundial da Saúde (2019). Who Global Report on Traditional and Complementary Medicine 2019. https://www.who.int/traditionalcomplementaryintegrativemedicine/WhoGlobalReportOnTraditionalAnd ComplementaryMedicine2019.pdf

Pereira, A. I. S. (2017). Atividade antibacteriana e caracterização físicoquímica de óleos essenciais extraídos das plantas medicinais comumente utilizadas pela população de São Luís do Maranhão [Tese de Doutorado, Universidade Estadual Paulista "Júlio de Mesquita Filho"]. Repositório Institucional UNESP. https://repositorio.unesp.br/handle/11449/150757

Pereira, M. A. A. (2010). Estudo da atividade antimicrobiana de óleos essenciais extraídos por destilação por arraste a vapor e por extração supercrítica [Dissertação de Mestrado, Pontifícia Universidade Católica do Rio Grande do Sul]. Repositório Institucional PUCRS. https://repositorio.pucrs.br/dspace/handle/10923/3383

Pinho, R. J. (2013). Efeito hepatoprotetor do óleo essencial de Alecrim e gengibre com pré-tratamento em modelo experimental de lesão induzida por paracetamol [Dissertação de Mestrado, Universidade Estadual de Maringá]. Repositório Institucional da Universidade Estadual de Maringá. http://repositorio.uem.br:8080/jspui/handle/1/2046

Pozzati, P. (2007). Suscetibilidade de Candida spp. resistentes e sensíveis ao fluconazol frente a óleos essenciais extraídos de condimentos [Dissertação de Mestrado, Universidade Federal de Santa Maria]. Repositório Digital da UFSM. https://repositorio.ufsm.br/handle/1/5993

Rafacho, B. P. M. (2015). Influência da suplementação de Alecrim (Rosmarinus oficinallis L.) na ração sobre a remodelação cardíaca em ratos submetidos ao infarto do miocárdio [Tese de Doutorado, Universidade Estadual Paulista "Júlio De Mesquita Filho". Repositório Institucional UNESP. https://repositorio.unesp.br/handle/11449/139321?locale-attribute=en

Ramos, R. S. (2014). Estudo fitoquímico e da atividade microbiológica, de citoxicidade e larvicida dos óleos essenciais de espécies da família Lamiaceae (Lamiales) [Dissertação de Mestrado, Universidade Federal do Amapá]. https://www2.unifap.br/ppgcf/files/2015/04/Dissertacao-Final_Ryan-da-SilvaRamos_04-12-2014.pdf

Rezende, D. A. C. S. (2016). Estudo etnobotânico de plantas medicinais de Lavras e região: caracterização química e potencialidades biológicas dos óleos essenciais [Dissertação de Mestrado, Universidade Federal de Lavras]. Repositório Institucional UFLA. http://repositorio.ufla.br/handle/1/11139

Rosa, J. S. (2013). Estudo do efeito anti-inflamatório da Rosmarinus Officinalis L. utilizando o modelo da pleurisia induzida pela carragenina em camundongos [Dissertação de Mestrado, Universidade Federal de Santa Catarina]. Repositório Institucional UFSC. https://repositorio.ufsc.br/handle/123456789/107141

Rubenick, J. B. (2017). Desenvolvimento de nanocápsulas contendo mupirocina e óleo essencial de Alecrim: caracterização e atividade antibiofilm [Dissertação de Mestrado, Universidade Federal de Santa Maria]. Repositório Digital da UFSM. https://repositorio.ufsm.br/handle/1/18411

Salmerón-Manzano, E, Garrido-Cardenas, J. A. \& Manzano-Agugliaro, F. (2020). Worldwide research trends on medicinal plants. International Jounal of Environmental Research and Public Health, 17(10), 3376. http://dx.doi.org/10.3390/ijerph17103376

Santos, L. D. (2017). Avaliação da toxicidade reprodutiva do óleo essencial de Rosmarinus officinalis l. em ratos wistar [Tese de Doutorado, Universidade Federal do Rio Grande do Sul]. Repositório Digital UFRGS. https://lume.ufrgs.br/handle/10183/163464 
Santos, U. D. (2013). Avaliação do potencial antinociceptivo e antiinflamatório do ácido rosmarínico [Tese de Doutorado, Universidade Federal de Santa Catarina]. Repositório Institucional UFSC. https://repositorio.ufsc.br/xmlui/handle/123456789/122777

Silva, A. M. O. (2012). Efeito dos compostos fenólicos do Alecrim (Rosmarinus officinalis L.) na inflamação aguda e sobre os marcadores de estresse oxidativo de ratos diabéticos [Tese de Doutorado, Universidade de São Paulo]. Biblioteca Digital USP. https://www.teses.usp.br/teses/disponiveis/9/9132/tde13032013-105302/pt-br.php

Silva, A. M. O. (2008). Efeito dos compostos fenólicos presentes no Alecrim (Rosmarinus officinalis L.) sobre as enzimas antioxidantes e os parâmetros bioquímicos do sangue de ratos diabéticos induzidos por estreptozotocina [Dissertação de Mestrado, Universidade de São Paulo]. Biblioteca Digital USP. https://teses.usp.br/teses/disponiveis/9/9132/tde-26092017-161242/pt-br.php

Silva, C. C. (2016). Estudo retrospectivo de melanomas cutâneos caninos e determinação da atividade citotóxica de produtos vegetais frente a células neoplásicas (B16F10) e não neoplásicas (MDBK) [Dissertação de Mestrado, Universidade Federal de Pelotas]. Guaiaca UFPel. http://guaiaca.ufpel.edu.br/handle/prefix/3599

Silva, G. K. F., Sousa, I. M. C. de, Cabral, M. E. G. da S., Bezerra, A. F. B. \& Guimarães, M. B. L. (2020). Política Nacional de Práticas Integrativas e Complementares: trajetória e desafios em 30 anos do SUS. Physis: Revista de Saúde Coletiva, 30(1), e300110. http://dx.doi.org/10.1590/S010373312020300110

Silva, M. S. A. (2005). Atividade antimicrobiana in vitro do extrato hidroalcoólico de Alecrim (Rosmarinus officinalis Linn.) sobre bactérias orais planctônicas [Dissertação de Mestrado em Odontologia, Universidade Federal de Pernambuco]. Repositório da UFPE. https://repositorio.ufpe.br/handle/123456789/8615

SILVA, N. F. (2018a). Avaliação da atividade antifúngica do óleo essencial de Rosmarinus officinalis L. sobre cepas dos dermatófitos Microsporum canis e Trichophyton rubrum [Dissertação de Mestrado, Universidade Federal do Acre]. Plataforma Sucupira. https://sucupira.capes.gov.br/sucu pira/public/consultas/coleta/trabalhoConclusao/viewTrabalhoConclusao.jsf?popup=true\&id_trabalho=7579912

SILVA, S. M. P. (2018b). Tratamento com ácido rosmarínico em parâmetro s bioquímico s e motores em modelo pré-clínico da Doença de Parkinson [Dissertação de Mestrado, Universidade Federal do Espírito Santo]. Repositório Ufes. http://repositorio.ufes.br/handle/10/7877

SILVA, T. G. (2019). Protótipo fitocosmético com potencial ação clareadora contendo extrato padronizado em ácido rosmarínico de Rosmarinus officinalis L [Dissertação de Mestrado, Universidade Federal de Goiás]. CFA UFG. https://sigaa.sistemas.ufg.br/sigaa/public/progr ama/noticias_desc.jsf? lc $=\mathrm{lc}=\mathrm{lc}=\mathrm{pt} \_$BR\&id=3199\&noticia $=970135353$

Takaki, I. (2006). Estudo das atividades antiinflamatória e antinociceptiva do óleo essencial de Alecrim (Rosmarinus officinalis L.) [Dissertação de Mestrado, Universidade Estadual de Maringá]. Portal Domínio Público. http://www.dominiopublico.gov.br/pesquisa/DetalheObraFormdo?select_a ction=\&co_obra=120643

Takayama, K. S. (2018). Efeito fotoquimiopreventivo de extrato de Rosmarinus officinalis sobre estresse oxidativo induzidos por radiação UVB em camundongos [Dissertação de Mestrado, Universidade Estadual de Londrina]. Biblioteca Digital UEL. http://www.bibliotecadigital.uel.br/document/?code=vtls000218599

Tenorio, L. A. (2014). Avaliação clínica da atividade do dentifrício à base do extrato de Rosmarinus Officinalis Linn. (Alecrim) sobre o biofilme dental-um estudo preliminar [Dissertação de Mestrado, Universidade Federal de Pernambuco]. Repositório Digital da UFPE. https://repositorio.ufpe.br/handle/123456789/12779

Tozatti, M. G. (2016). Busca por substâncias naturais com potencial aplicação frente a bactérias resistentes [Tese de Doutorado, Universidade de Franca]. Plataforma Sucupira. https://sucupira.capes.gov.br/sucupira/public/consultas/coleta/trabalhoConclusao/viewTrabalhoConclusao.jsf?popup=true\&id_ trabalho $=3779836$

Valones, M. A. A. (2016). Avaliação clínica de um dentifrício a base do extrato DE Rosmarinus officinalis Linn., quanto ao sangramento gengival e presença de placa bacteriana através de ensaio clínico randomizado controlado duplo-cego [Tese de Doutorado, Universidade Federal de Pernambuco. Repositório Digital da UFPE. https://repositorio.ufpe.br/handle/123456789/26606

Valones, M. A. A. (2008). Avaliação da atividade antimicrobiana in vitro do dentifrício à base do extrato alcoólico de Rosmarinus officinalis Linn. (Alecrim) sobre cepas padrão de S. mutans, S. aureus e L. casei [Dissertação de Mestrado, Universidade Federal de Pernambuco. Repositório Digital da UFPE. https://repositorio.ufpe.br/handle/123456789/8053

Wanderley, A. L. (2015). Atividade antioxidante e antimicrobiana do óleo essencial de Rosmainus Officinalis l., cultivado em sistema orgânico sob diferentes condições, frente a bactérias causadoras de mastite bovina [Dissertação de Mestrado, Universidade de Brasília]. Repositório Institucional da UNB. https://repositorio.unb.br/handle/10482/19398

Waller, S. B. (2015). Potencial anti-Sporothrix spp. de plantas da família Lamiaceae [Dissertação de Mestrado, Universidade Federal do Rio Grande do Sul. Repositório Digital UFRGS. https://lume.ufrgs.br/handle/10183/118276

Warken, M. N. (2016). Avaliação da atividade cicatrizante de uma emulsão contendo óleo de Rosmarinus officinalis [Dissertação de Mestrado, Universidade Luterana do Brasil]. Espaço Digital uepb. http://dspace.bc.uepb.edu.br/jspui/bitstream/123456789/6803/1/PDF\%20\%20Gabriela\%20Baptista\%20de\% 20Assis.pdf

Whittemore, R., \& Knafl, K. (2005). The integrative review: updated methodology. Journal of Advanced Nursing, 52(5), 546-553. https://pubmed.ncbi.nlm.nih.gov/16268861/

Yamamoto, I. M. de P. (2013). Rosmarinus officinalis Linn avaliando o potencial para preparação de enxaguatório bucal [Dissertação de Mestrado, Universidade Federal de Juiz de Fora]. Repositório Institucional UFJF. https://repositorio.ufjf.br/jspui/handle/ufjf/2379 
Research, Society and Development, v. 10, n. 9, e50910918255, 2021

(CC BY 4.0) | ISSN 2525-3409 | DOI: http://dx.doi.org/10.33448/rsd-v10i9.18255

Zamberlan, D. C. (2018). Efeito de compostos naturais no envelhecimento e em modelos de doença de Alzheimer no nematódeo Caernorhabditis elegans [Tese de Doutorado, Universidade Federal de Santa Maria]. Repositório Digital da UFSM. https://repositorio.ufsm.br/handle/1/16712

Zibetti, F. M. (2016). Desenvolvimento de formulação nanoestruturada contendo óleo essencial de Rosmarinus officinalis L. para o tratamento tópico do herpes [Dissertação de Mestrado, Universidade Federal Fluminense]. Repositório Institucional da Universidade Federal Fluminense. https://app.uff.br/riuff/handle/1/5480 